\title{
Impact of Enhanced Recovery After Surgery on Long-Term Outcomes and Postoperative Recovery in Patients Undergoing Hepatectomy: A Retrospective Cohort Study
}

This article was published in the following Dove Press journal:

Cancer Management and Research

\author{
Zhao Zhang' \\ Weidong $\mathrm{Gu}^{2}$ \\ Yijing Zhang $\mathbb{D}^{2}$ \\ 'Nursing Department, Huadong Hospital \\ Affiliated to Fudan University, Shanghai, \\ 200040, People's Republic of China; \\ ${ }^{2}$ Department of Anesthesiology, \\ Huadong Hospital Affiliated to Fudan \\ University, Shanghai, 200040, People's \\ Republic of China
}

Background: The aim of this study was to evaluate the effects of implementation of the enhanced recovery after surgery (ERAS) program on postoperative recovery and the longterm prognosis in patients who underwent hepatectomy.

Methods: This retrospective study enrolled patients who underwent hepatectomy from January 2015 to December 2018 in Huadong Hospital Affiliated to Fudan University. Since June 2016, a 24-point ERAS protocol has been implemented for patients who underwent hepatic resection. The primary outcomes were overall survival (OS) and disease-free survival (DFS). The secondary outcomes included length of stay (LOS), and incidence of postoperative complications.

Results: A total of 1143 patients were enrolled in this study. After propensity score matching (PSM), there was no significant difference in patients' demographic characteristics. The DFS at 1., 3 years in ERAS group was higher than in non-ERAS group ( $96.3 \%$ vs $88.9 \%$ for 1 year, $\mathrm{P}=0.012 ; 58.9 \%$ vs $46.7 \%$ for 3 years, $\mathrm{P}=0.007$ ). The OS at 1,3 years in ERAS group was higher than in non-ERAS group $(93.1 \%$ vs $89.3 \%$ for 1 year, $\mathrm{P}=0.041 ; 68.7 \%$ vs $61.2 \%$ for 3 years, $\mathrm{P}=0.035$ ). In addition, the patients in ERAS group had lower incidences of postoperative hemorrhage, bile leak, and postoperative deep vein thrombosis/pulmonary embolism (DVT/PE), decreased 30-day readmission rate and total readmission rate, and shorter LOS.

Conclusion: ERAS program could be safely applied to patients who underwent hepatectomy thereby improving their recovery and prolonging OS and DFS.

Keywords: hepatectomy, enhanced recovery after surgery, length of stay, overall survival, disease-free survival

\section{Introduction}

Liver cancer is the sixth most common cancer, and the fourth leading cause of cancer-related deaths worldwide. ${ }^{1}$ Currently, hepatectomy is still the preferred treatment for early and intermediate stage liver cancer. ${ }^{2}$ However, as a major abdominal operation of great difficulty and complexity, hepatectomy has a high incidence of complications. Studies have shown that the perioperative period plays a critical role in cancer progression. ${ }^{3}$ Perioperative management of hepatectomy has gradually attracted attention, to improve the long-term prognosis of patients with liver cancer.
Correspondence: Yijing Zhang

Department of Anesthesiology, Huadong Hospital Affiliated to Fudan University, No. 22I West Yan'an Road, Shanghai, 200040, People's Republic of China Email ZI3901683755@163.com 
Enhanced recovery after surgery (ERAS) was first introduced in 1997, ${ }^{4}$ and is a series of perioperative optimization measures based on evidence-based medicine, aiming to promote patients' recovery, shorten hospital stay, reduce postoperative complications, and lessen health care costs. ${ }^{5}$ The ERAS protocol has been widely implemented in gastrointestinal surgery, prostatectomy, orthopedics surgery, and other fields and has achieved remarkable results. ${ }^{6-8}$ The ERAS protocol applied in patients undergoing minimally invasive radical prostatectomy could shorten length of stay (LOS) and reduced hospitalization costs. $^{9}$ The introduction of ERAS for anterior cervical discectomy and fusion significantly decreased the LOS, without causing an increase in postoperative complications. ${ }^{10}$ ERAS protocol decreases surgical stress and accelerates functional recovery in patients undergoing colorectal surgery. ${ }^{11,12}$ Due to the complexity and high risk of liver surgery, the application of ERAS in hepatectomy is still in the exploratory stage. Studies have shown that ERAS significantly shortens the length of patient hospitalizations, accelerates the rehabilitation process, reduces readmission rates and medical costs in patients undergoing hepatectomy surgery. ${ }^{13}$ However, there have been few studies about the effect of ERAS on the long-term prognosis in patients who underwent hepatectomy.

In this study, we hypothesized that the implementation of ERAS protocol is an independent predictor of improved survival outcomes in patients undergoing hepatectomy. We also studied the associations between the implementation of ERAS protocol and incidence of postoperative complications, 90-day mortality, 30-day and 90-day readmission rates, and LOS.

\section{Methods}

\section{Study Population}

This study was conducted in accordance with the Declaration of Helsinki Ethical Principles, and approved by the Ethics Committee of Huadong Hospital Affiliated to Fudan University. All patients enrolled in the analysis signed a consent for data used for research before receiving treatment. From January 2015 to December 2017, patients who underwent hepatectomy for liver cancer were enrolled in this retrospective cohort. The exclusion criteria included: a) emergency surgery; b) patients with cognitive dysfunction and other difficulties in cooperation; c) severe chronic disease including cardiac and pulmonary disease; d) coexistence of other tumors; e) incomplete information and loss of contact during follow-up; f) did not die due to serious postoperative complications. Data were collected from the database of the clinical information system. The medical information of each patient was reviewed and recorded, which included demographic information, medical history, primary diagnosis, operative details, tumor differentiation and pathological staging, and DFS and OS time.

\section{Primary Outcomes}

We assessed primary outcomes (DFS and OS) in the patients after ERAS implementation. DFS was defined as the length of time from the date of surgery to the date of the first evidence of tumor recurrence or to December 31, 2019. OS was defined as the length of time from the date of surgery to the date of death or the last follow-up date.

\section{Secondary Outcomes}

To evaluate the safety and efficacy of the ERAS protocol, the secondary outcomes were the postoperative LOS, readmission rates at 30 and 90 days, and incidence of postoperative complications. The LOS was defined as the number of nights in the hospital postoperatively. Data on patients' readmission on day 30 and 90 were collected. Postoperative complications included postoperative hemorrhage, vomiting, post-hepatectomy liver failure (PHLF), bile leak, and postoperative deep vein thrombosis/pulmonary embolism (DVT/PE).

\section{ERAS Protocol}

Since June 2016, a 24-point ERAS protocol has been implemented for patients who underwent elective hepatic resection. Based on expert consensus and guidelines of ERAS, patients in ERAS group received perioperative treatment under the guidance of ERAS concepts. Patients treated before June 2016 were classified as the non-ERAS group and received conventional perioperative treatment; patients treated after June 2016 were classified as the ERAS group and received the standard ERAS protocol treatment of Huadong Hospital Affiliated to Fudan University. Within the time period of this study, the surgical staff, consisting of 10 experienced surgeons, anesthesiologists, and nursing staff, remained basically unchanged. The two groups of specific programs are shown in Table 1 .

\section{Statistical Analysis}

Continuous variables were summarized as mean or as median and were compared by $t$-test and ANOVA. Categorical variables summarized by percentages were 
Table I Summary of the ERAS Protocols and Comparison with Conventional Perioperative Management

\begin{tabular}{|c|c|c|}
\hline Period & ERAS Group & Non-ERAS Group \\
\hline $\begin{array}{l}\text { Preoperative } \\
\text { period }\end{array}$ & $\begin{array}{l}\text { Preoperative counseling: advised by surgeon, anesthesiologist, and nurse about the } \\
\text { surgery and anesthesia type and perioperative risk } \\
\text { Written detailed recovery plan } \\
\text { Perioperative nutrition: surgery should be postponed to improve nutritional status } \\
\text { when diagnosed with body weight loss } 10-15 \% \text { in } 6 \text { months, BMl< } 18.5 \mathrm{~kg} / \mathrm{m} 2 \text { and serum } \\
\text { albumin }<30 \mathrm{~g} / \mathrm{L} \\
\text { Preoperative fasting and preoperative carbohydrates load: allow clear liquid until } 2 \mathrm{~h} \\
\text { before surgery } \\
\text { Allow solid foods until } 6 \mathrm{~h} \text { before surgery } \\
\text { Oral carbohydrate solution loading over two days prior to surgery } \\
\text { Anti-thrombotic prophylaxis: wear compression stockings before surgery } \\
\text { Optimization of comorbidities } \\
\text { Smoking and alcohol advice } \\
\text { Daily exercise: walk I hour per day between first consultation and surgery } \\
\text { Preoperative bowel preparation } \\
\text { No routine mechanical bowel preparation }\end{array}$ & $\begin{array}{l}\text { Advised by a surgeon about surgery } \\
\text { risk } \\
\text { None } \\
\text { Fasting/no solid food } 8 \text { hours before } \\
\text { surgery } \\
\text { Fasting/liquid } 4 \text { hours before surgery } \\
\text { None } \\
\text { No strict requirements } \\
\text { No strict requirements } \\
\text { Routine mechanical bowel preparation }\end{array}$ \\
\hline $\begin{array}{l}\text { Intraoperative } \\
\text { period }\end{array}$ & $\begin{array}{l}\text { Pre-anesthetic medication: short-acting anxiolytics used to relieve anxiety prior to the } \\
\text { induction of anesthesia (dexmedetomidine) } \\
\text { Anesthesia: total intravenous anesthesia was used } \\
\text { Optimization of fluid balance by target-directed fluid therapy } \\
\text { Preventing intraoperative hypothermia } \\
\text { Active warming with a warming blanket } \\
\text { Warm intravenous fluid with a liquid warming tube } \\
\text { Mini incision: surgical incision depends on the patient's abdominal shape and tumor } \\
\text { location in the liver. Mercedes-type incision should be avoided due to higher incisional } \\
\text { hernia risk } \\
\text { Prevention of delayed gastric emptying (DGE): the omentum flap should cover the cut } \\
\text { surface of the liver to reduce the risk of DGE after left-sided hepatectomy } \\
\text { Perioperative steroids administration: steroids used before hepatectomy in normal liver } \\
\text { parenchyma and not given in diabetic patients } \\
\text { Antimicrobial prophylaxis and skin preparation: single dose intravenous antibiotics } \\
\text { should be administered before skin incision and less than I h before hepatectomy }\end{array}$ & $\begin{array}{l}\text { Dependent on the accessibility for the } \\
\text { surgeon } \\
\text { None } \\
\text { None } \\
\text { Depends on the risk of postoperative } \\
\text { infection risk }\end{array}$ \\
\hline $\begin{array}{l}\text { Postoperative } \\
\text { period }\end{array}$ & $\begin{array}{l}\text { Postoperative analgesia: comprehensive, quantitative, and dynamic evaluation combined } \\
\text { with preventive multi-mode analgesic management (eg, PCEA +NSAIDs +wound } \\
\text { infusion) } \\
\text { Prophylactic nasogastric intubation: not used } \\
\text { Preventing postoperative nausea and vomiting (PONV): multimodal approach to PONV } \\
\text { should be used } \\
\text { Early mobilization: should be encouraged from the morning after the operation until } \\
\text { hospital discharge: walk } 10-20 \mathrm{~m} \text { on PODI, walk } 100 \mathrm{~m} \text { on POD2, walk } 100 \mathrm{~m}+\text { on } \\
\text { POD3 } \\
\text { Stimulation of bowel movement: chewing gum encouraged to stimulate gut motility } \\
\text { Postoperative glycemic control: insulin therapy to maintain normoglycemia is } \\
\text { recommended } \\
\text { Consider removal of pelvic drainage as early as possible } \\
\text { Audit: systematic audit improves compliance and clinical outcome in healthcare } \\
\text { practice }\end{array}$ & $\begin{array}{l}\text { Selective on-demand/symptomatic } \\
\text { treatment } \\
\text { Depends on surgery risk } \\
\text { Selective on-demand/symptomatic } \\
\text { treatment } \\
\text { Mobilization was started at } 2-3 \text { d after } \\
\text { surgery } \\
\text { Individualized liver blood flow control } \\
\text { technique } \\
\text { Individualized liver blood flow control } \\
\text { technique } \\
\text { Care decided by the surgeon } \\
\text { None }\end{array}$ \\
\hline
\end{tabular}


compared by the Chi-squared test. Covariables included in the calculation of propensity score included gender, age, liver cirrhosis, cirrhosis, tumor encapsulation, alpha fetoprotein (AFP), tumor size, tumor staging, tumor number, vascular invasion, American Standards Association (ASA) grading, and surgical procedure. In order to reduce bias, propensity score matching was used. Patients were matched using a 5-to-1 digit Greedy match algorithm. The DFS and OS were compared using the Kaplan-Meier method. Univariate analyses were fitted to compare differences between patients with and without ERAS implementation using the $\chi 2$ test for categorical variables and Wilcoxon rank-sum test for continuous variables. Multivariable analysis of Cox model was conducted to identify important and significant covariates. SPSS 24.0 (SPSS Inc., Chicago, IL, USA) was used for the analysis of propensity score matching and other statistical analyses. An $\alpha$ value of 0.05 was used as a threshold for statistical significance.

\section{Results}

A total of 1143 patients were enrolled in this study, after exclusion, 1095 patients were enrolled in this study
(Figure 1). Of these, 463 patients who underwent surgery were included in ERAS programs, and 632 patients who underwent surgery were included in non-ERAS programs. Clinical characteristics comparing ERAS group and nonERAS group were shown in Table 2. After propensity score matching, there were 463 patients left in each group.

\section{Primary Outcome}

In this study, the median follow-up time for all patients was 36.5 months $(95 \% \mathrm{CI}, 33.7,42.0)$. The median follow-up time for patients in ERAS group was 35.8 months $(95 \% \mathrm{CI}, 34.7,41.5)$ and in the non-ERAS group was 37.3 (95\% CI, 35.7, 42.0) months. The Kaplan Meier survival curves for the ERAS group and non-ERAS group are displayed in Figure 2A. The DFS rates at 1 and 3 years after surgery were different between patients in the ERAS group and those in the non-ERAS group (96.3\% vs $88.9 \%$ for $1 \mathrm{y}, \mathrm{P}=0.012 ; 58.9 \%$ vs $46.7 \%$ for $3 \mathrm{y}, \mathrm{P}=0.007$, respectively Figure $2 \mathrm{~A}$ ). Univariate analysis for factors contributing to DFS was presented in Table 3. Factors found to be independently predictive of DFS were ERAS protocol $(\mathrm{P}<0.001)$, age $(\mathrm{P}=0.01)$, AFP $(\mathrm{P}=0.026)$, tumor staging (III-IV) $(\mathrm{P}=0.022)$, vascular invasion $(\mathrm{P}<0.003)$,

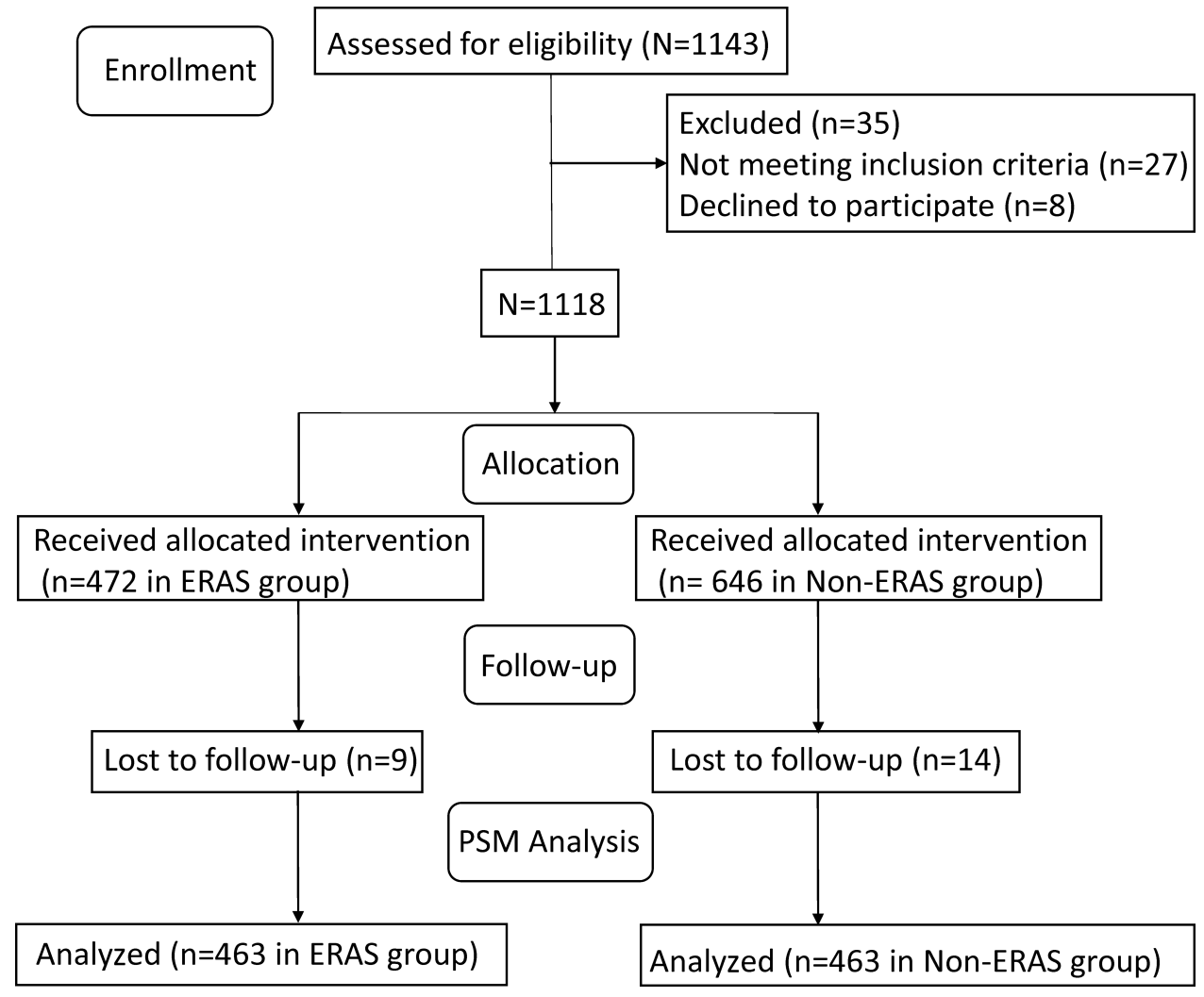

Figure I Flow diagram detailing the selection process for patients included in this retrospective analysis. 
Table 2 Clinical Characteristics of the Patients Undergoing Hepatectomy Between ERAS Group and Non-ERAS Group

\begin{tabular}{|c|c|c|c|c|c|c|}
\hline \multirow[t]{2}{*}{ Variable } & \multicolumn{2}{|c|}{ Original Cohort } & \multirow[t]{2}{*}{$P$} & \multicolumn{2}{|c|}{ Matched Cohort } & \multirow[t]{2}{*}{$P$} \\
\hline & $\begin{array}{l}\text { ERAS Group } \\
\qquad(n=463)\end{array}$ & $\begin{array}{l}\text { Non-ERAS Group } \\
(n=632)\end{array}$ & & $\begin{array}{l}\text { ERAS Group } \\
\qquad(n=463)\end{array}$ & $\begin{array}{l}\text { Non-ERAS Group } \\
\qquad(n=463)\end{array}$ & \\
\hline Age (years) & $63.2 \pm 8.6$ & $62.8 \pm 9.2$ & 0.465 & $63.2 \pm 8.6$ & $62.8 \pm 9.2$ & 0.495 \\
\hline $\begin{array}{l}\text { Sex }(n, \%) \\
\text { Female } \\
\text { Male }\end{array}$ & $\begin{array}{l}72(15.6 \%) \\
391(84.4 \%)\end{array}$ & $\begin{array}{c}96(\mid 14.8 \%) \\
536(85.2 \%)\end{array}$ & 0.870 & $\begin{array}{c}74(15.6 \%) \\
389(84.4 \%)\end{array}$ & $\begin{array}{c}70(15.6 \%) \\
393(84.4 \%)\end{array}$ & 0.786 \\
\hline BMI kg/m2, (median-IQR) & $22.3(21.5-25.4)$ & $23.6(21.7-25.6)$ & & $22.5(21.6-25.2)$ & $23.6(21.7-25.6)$ & \\
\hline $\begin{array}{l}\text { ASA }(n, \%) \\
\text { I } \\
\text { II } \\
\text { III }\end{array}$ & $\begin{array}{c}32(6.9 \%) \\
395(85.3 \%) \\
36(7.8 \%)\end{array}$ & $\begin{array}{c}39(6.2 \%) \\
528(83.6 \%) \\
65(10.2 \%)\end{array}$ & $0.34 I$ & $\begin{array}{c}32(6.9 \%) \\
395(85.3 \%) \\
36(7.8 \%)\end{array}$ & $\begin{array}{c}32(6.9 \%) \\
396(85.3 \%) \\
35(7.8 \%)\end{array}$ & 0.992 \\
\hline $\begin{array}{l}\text { Patients enrolled (n, \%) } \\
2014 \\
2015 \\
2016 \\
2017\end{array}$ & $\begin{array}{l}112(24.2 \%) \\
106(22.9 \%) \\
125(27.0 \%) \\
120(25.9 \%)\end{array}$ & $\begin{array}{l}149(23.6 \%) \\
139(22.0 \%) \\
176(27.8 \%) \\
168(26.6 \%)\end{array}$ & 0.968 & $\begin{array}{l}112(24.2 \%) \\
106(22.9 \%) \\
125(27.0 \%) \\
120(25.9 \%)\end{array}$ & $\begin{array}{l}112(24.2 \%) \\
104(22.9 \%) \\
127(27.0 \%) \\
120(25.9 \%)\end{array}$ & 0.998 \\
\hline $\begin{array}{l}\mathrm{CCl}(\mathrm{n}, \%) \\
0 \\
\mathrm{l} \\
\geqq 2\end{array}$ & $\begin{array}{l}256(55.3 \%) \\
132(28.5 \%) \\
75(16.2 \%)\end{array}$ & $\begin{array}{l}345(54.6 \%) \\
166(26.3 \%) \\
121(19.1 \%) \\
\end{array}$ & 0.404 & $\begin{array}{l}256(55.3 \%) \\
132(28.5 \%) \\
75(16.2 \%) \\
\end{array}$ & $\begin{array}{l}254(55.3 \%) \\
134(28.5 \%) \\
75(16.2 \%) \\
\end{array}$ & 0.989 \\
\hline $\begin{array}{l}\text { Hepatitis B surface antigen } \\
\text { (n, \%) } \\
\quad \text { Negative } \\
\text { Positive }\end{array}$ & $\begin{array}{l}160(34.6 \%) \\
303(65.4 \%)\end{array}$ & $\begin{array}{l}228(36.1 \%) \\
404(63.9 \%)\end{array}$ & 0.604 & $\begin{array}{l}160(34.6 \%) \\
303(65.4 \%)\end{array}$ & $\begin{array}{l}159(34.6 \%) \\
304(65.4 \%)\end{array}$ & 0.945 \\
\hline $\begin{array}{l}\text { Child-Pugh classification } \\
\text { (n, \%) } \\
\text { A } \\
\text { B }\end{array}$ & $\begin{array}{l}412(88.9 \%) \\
51(11.1 \%)\end{array}$ & $\begin{array}{l}551(87.2 \%) \\
81(12.8 \%)\end{array}$ & 0.366 & $\begin{array}{l}412(88.9 \%) \\
51(11.1 \%)\end{array}$ & $\begin{array}{c}410(88.9 \%) \\
53(11.1 \%)\end{array}$ & 0.835 \\
\hline $\begin{array}{l}\text { Serum AFP (ug/L) } \\
\quad \leq 20 \\
>20\end{array}$ & $\begin{array}{l}74(15.9 \%) \\
389(84.1 \%)\end{array}$ & $\begin{array}{l}89(14.1 \%) \\
543(85.9 \%)\end{array}$ & 0.383 & $\begin{array}{l}74(15.9 \%) \\
389(84.1 \%)\end{array}$ & $\begin{array}{l}73(15.9 \%) \\
390(84.1 \%)\end{array}$ & 0.928 \\
\hline $\begin{array}{l}\text { Tumor size (n, \%) } \\
\quad \leq 5 \mathrm{~cm} \\
>5 \mathrm{~cm}\end{array}$ & $\begin{array}{l}215(46.4 \%) \\
248(53.6 \%)\end{array}$ & $\begin{array}{l}286(45.2 \%) \\
346(54.8 \%)\end{array}$ & 0.698 & $\begin{array}{l}215(46.4 \%) \\
248(53.6 \%)\end{array}$ & $\begin{array}{l}213(46.4 \%) \\
250(53.6 \%)\end{array}$ & 0.895 \\
\hline $\begin{array}{l}\text { Tumor number (n, \%) } \\
\text { Single } \\
\text { Multiple }\end{array}$ & $\begin{array}{c}393(84.9 \%) \\
70(15.1 \%)\end{array}$ & $\begin{array}{l}525(83.2 \%) \\
107(16.8 \%)\end{array}$ & 0.421 & $\begin{array}{c}393(84.9 \%) \\
70(15.1 \%)\end{array}$ & $\begin{array}{l}391(84.9 \%) \\
72(15.1 \%)\end{array}$ & 0.855 \\
\hline $\begin{array}{l}\text { Tumor encapsulation (n, \%) } \\
\text { No/incomplete } \\
\text { Yes }\end{array}$ & $\begin{array}{l}376(81.2 \%) \\
87(18.8 \%)\end{array}$ & $\begin{array}{l}525(83.1 \%) \\
107(16.9 \%)\end{array}$ & 0.426 & $\begin{array}{l}376(81.2 \%) \\
87(18.8 \%)\end{array}$ & $\begin{array}{c}374(81.2 \%) \\
89(18.8 \%)\end{array}$ & 0.867 \\
\hline $\begin{array}{l}\text { Vascular invasion (n, \%) } \\
\text { No } \\
\text { Yes }\end{array}$ & $\begin{array}{c}405(87.5 \%) \\
58(12.5 \%)\end{array}$ & $\begin{array}{c}544(86.1 \%) \\
88(13.9 \%)\end{array}$ & 0.502 & $\begin{array}{c}405(87.5 \%) \\
58(12.5 \%)\end{array}$ & $\begin{array}{c}402(87.5 \%) \\
61(12.5 \%)\end{array}$ & 0.768 \\
\hline
\end{tabular}


Table 2 (Continued).

\begin{tabular}{|c|c|c|c|c|c|c|}
\hline \multirow[t]{2}{*}{ Variable } & \multicolumn{2}{|c|}{ Original Cohort } & \multirow[t]{2}{*}{$\boldsymbol{P}$} & \multicolumn{2}{|c|}{ Matched Cohort } & \multirow[t]{2}{*}{$P$} \\
\hline & $\begin{array}{l}\text { ERAS Group } \\
(n=463)\end{array}$ & $\begin{array}{l}\text { Non-ERAS Group } \\
\qquad(n=632)\end{array}$ & & $\begin{array}{l}\text { ERAS Group } \\
\quad(n=463)\end{array}$ & $\begin{array}{l}\text { Non-ERAS Group } \\
\qquad(n=463)\end{array}$ & \\
\hline Liver cirrhosis (n, \%) & & & 0.617 & & & 0.997 \\
\hline No & $42(9.0 \%)$ & $5 \mathrm{I}(8.1 \%)$ & & $42(9.0 \%)$ & $42(9.0 \%)$ & \\
\hline Mild & $256(55.3 \%)$ & $347(54.9 \%)$ & & $256(55.3 \%)$ & $253(55.3 \%)$ & \\
\hline Moderate & $83(17.9 \%)$ & $104(16.4 \%)$ & & $83(17.9 \%)$ & $84(17.9 \%)$ & \\
\hline Severe & $82(17.8 \%)$ & $130(20.6 \%)$ & & $82(17.8 \%)$ & $84(17.8 \%)$ & \\
\hline BCLC stage $(n, \%)$ & & & 0.335 & & & 0.989 \\
\hline 0 & $31(6.7 \%)$ & $39(6.1 \%)$ & & $31(6.7 \%)$ & $31(6.7 \%)$ & \\
\hline A & $409(88.3 \%)$ & $548(86.7 \%)$ & & $409(88.3 \%)$ & $408(88.3 \%)$ & \\
\hline B & $23(5.0 \%)$ & $45(5.0 \%)$ & & $23(5.0 \%)$ & $24(5.0 \%)$ & \\
\hline Estimated blood loss (n, \%) & & & 0.665 & & & 0.944 \\
\hline$\leq 400 \mathrm{~mL}$ & $312(67.4 \%)$ & $418(66.2 \%)$ & & $312(67.4 \%)$ & $313(67.4 \%)$ & \\
\hline$>400 \mathrm{~mL}$ & $|5|(32.6 \%)$ & $214(33.8 \%)$ & & $15 \mid(32.6 \%)$ & $150(32.6 \%)$ & \\
\hline Blood transfusion $(n, \%)$ & & & 0.785 & & & 0.932 \\
\hline No & $381(82.3 \%)$ & $516(81.6 \%)$ & & $38 I(82.3 \%)$ & $380(82.3 \%)$ & \\
\hline Yes & $82(17.7 \%)$ & $116(18.4 \%)$ & & $82(17.7 \%)$ & $83(17.7 \%)$ & \\
\hline Pathology diagnosis (n, \%) & & & 0.091 & & & 0.979 \\
\hline $\mathrm{HCC}$ & $376(81.2 \%)$ & $509(80.6 \%)$ & & $376(81.2 \%)$ & $374(81.2 \%)$ & \\
\hline ICC & $53(11.4 \%)$ & $58(9.4 \%)$ & & $53(\mathrm{II.4 \% )}$ & $55(11.4 \%)$ & \\
\hline $\mathrm{CHC}$ & $32(7.4 \%)$ & $65(10 \%)$ & & $32(7.4 \%)$ & $32(7.4 \%)$ & \\
\hline Operative methods (n, \%) & & & 0.618 & & & 0.860 \\
\hline Open & $101(21.8 \%)$ & $130(20.6 \%)$ & & $101(21.8 \%)$ & $103(21.8 \%)$ & \\
\hline Laparoscopic & $362(78.2 \%)$ & $502(79.4 \%)$ & & $362(78.2 \%)$ & $360(78.2 \%)$ & \\
\hline Anesthesia method (n, \%) & & & 0.309 & & & 0.895 \\
\hline GA & $31(6.7 \%)$ & $40(6.3 \%)$ & & $31(6.7 \%)$ & $30(6.7 \%)$ & \\
\hline $\mathrm{GA}+\mathrm{GEA}$ & $432(93.3 \%)$ & $592(93.7 \%)$ & & $432(93.3 \%)$ & $433(93.3 \%)$ & \\
\hline
\end{tabular}

Abbreviations: HCC, hepatocellular carcinoma; ICC, cholangiocarcinoma; CHC, combined hepatocellular carcinoma and cholangiocarcinoma.

and ASA (III-IV) $(\mathrm{P}=0.021)$. The multivariable logistic regression model showed that ERAS $(\mathrm{P}<0.001)$, age $(\mathrm{P}=0.006)$, vascular invasion ( $\mathrm{P}=0.002)$, and ASA (IIIIV) $(\mathrm{P}=0.045)$, were associated with $\mathrm{OS}$ in the original cohort. After PSM, ERAS protocol was still found to be independently predictive of $\mathrm{OS}([\mathrm{CI}] 1.32-2.62 ; \mathrm{P}=0.016$ ) (Table 4).

The OS of patients who underwent hepatectomy for liver cancer in the ERAS group was significantly higher compared with non-ERAS group $(93.1 \%$ vs $89.3 \%$ for $1 \mathrm{y}$, $\mathrm{P}=0.041 ; 68.7 \%$ vs. $61.2 \%$ for $3 \mathrm{y}, \mathrm{P}=0.035$, respectively, Figure 2B). Univariate analysis for factors contributing to OS was presented in Table 3. Factors found to be independently predictive of $\mathrm{OS}$ were age $(\mathrm{P}=0.03)$, AFP $(\mathrm{P}=0.011)$, tumor encapsulation $(\mathrm{P}=0.044)$, vascular invasion $\quad(\mathrm{P}<0.001)$, and ASA (III-IV) $\quad(\mathrm{P}=0.013)$.
Multivariable analysis for factors contributing to OS was presented in Table 4. Age $(\mathrm{P}=0.018)$, AFP $(\mathrm{P}=0.022)$, vascular invasion $(\mathrm{P}=0.004)$, and ASA (III-IV) $(\mathrm{P}=0.039)$ were associated with $\mathrm{OS}$ in the original cohort. After PSM, ERAS protocol was still found to be independently predictive of OS ([CI] 1.02-2.12; $\mathrm{P}=0.013$ ) (Table 4).

\section{Secondary Outcome}

Patients in ERAS group had lower 30-day readmission rate and total readmission rate than those in non-ERAS group (Figure 3A). Hospital LOS was significantly shorter in the ERAS group at 10.4 days compared with 12.3 days in the non-ERAS group $(\mathrm{P}<0.05)$ (Figure $3 \mathrm{~B})$. In addition, the Clavien-dindo classification showed significant difference between the two groups (Figure 3C). Compared with the 
A

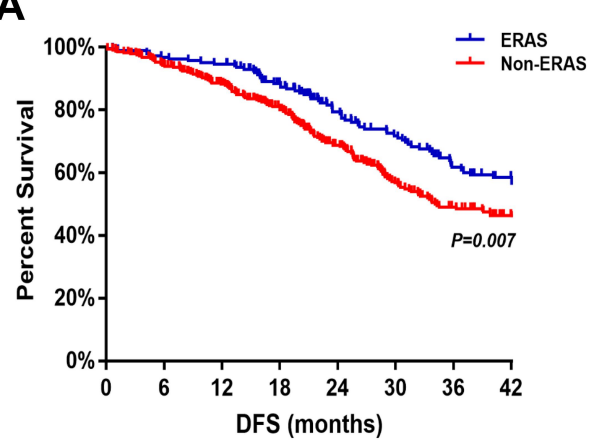

B

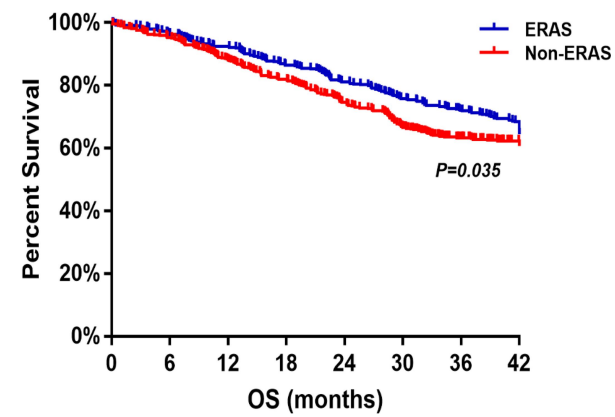

Figure 2 (A) Disease-free survival curves from the date of surgery between ERAS group and non-ERAS group; (B) overall survival curves from the date of surgery between ERAS group and non-ERAS group.

Abbreviations: DFS, disease-free survival; OS, overall survival.

non-ERAS group, the incidences of overall complications (including postoperative hemorrhage, PHLF, bile leak, and postoperative DVT/PE) were significantly lower in the ERAS group (Figure 3D).

\section{Discussion}

Liver cancer is the sixth most prevailing cancer worldwide. ${ }^{14}$ Benefitting from the advances of liver surgery technology, the treatment of liver cancer has changed from the goal of pursuing a longer survival time to the goal of minimizing traumatic stress and promoting rapid recovery on the premise of ensuring radical treatment and improving overall survival.

An ERAS program integrates the perioperative optimal measures that are based on medical evidence, aiming to reduce surgical trauma and stress, promote adequate

Table 3 Univariate Analysis of OS and DFS

\begin{tabular}{|c|c|c|c|c|}
\hline \multirow[t]{2}{*}{ Variables } & \multicolumn{2}{|c|}{ os } & \multicolumn{2}{|c|}{ DFS } \\
\hline & HR (95\% Cl) & $P$-value & HR (95\% CI) & $P$-value \\
\hline ERAS Protocol (no vs yes) & $1.60(1.03,3.73)$ & $<0.001$ & $1.34(1.27,2.60)$ & $<0.001$ \\
\hline Age (years) & $1.07(1.01,1.14)$ & 0.030 & $1.10(1.03,1.18)$ & 0.010 \\
\hline Gender (male) & $0.63(0.38,5.15)$ & 0.665 & $0.92(0.53,1.59)$ & 0.764 \\
\hline Liver cirrhosis (Yes) & $0.70(0.39, I .27)$ & 0.237 & $0.17(0.03,1.17)$ & 0.072 \\
\hline AFP $(>20 \mathrm{ng} / \mathrm{mL})$ & $1.69(1.13,2.53)$ & 0.011 & $1.44(1.04,1.98)$ & 0.026 \\
\hline Tumor encapsulation (None) & $2.36(1.02,5.42)$ & 0.044 & $1.55(0.90,2.67)$ & 0.115 \\
\hline Tumor staging (III-IV) & $1.80(1.66,3.35)$ & 0.066 & $1.47(1.06,2.04)$ & 0.022 \\
\hline Tumor size $(>5 \mathrm{~cm})$ & $1.39(0.72,2.70)$ & 0.323 & $1.20(0.78,1.83)$ & 0.410 \\
\hline Tumor number (Multiple) & $1.22(0.72,2.06)$ & 0.462 & $1.37(0.91,2.08)$ & 0.132 \\
\hline Vascular invasion (Yes) & $3.45(1.83,6.53)$ & $<0.001$ & $2.38(1.34,4.21)$ & 0.003 \\
\hline ASA (III-IV) & $1.89(1.14,3.14)$ & 0.013 & $2.77(1.17,6.54)$ & 0.021 \\
\hline Surgical procedure (Video-assisted) & $1.10(0.41,2.97)$ & 0.845 & $1.34(0.52,3.41)$ & 0.545 \\
\hline Blood loss (>400mL) & $1.35(0.52,3.52)$ & 0.538 & $1.09(0.63,1.90)$ & 0.751 \\
\hline Blood transfusion (yes) & $1.26(0.90,1.42)$ & 0.234 & $1.45(0.82,1.62)$ & 0.651 \\
\hline Postoperative complication (yes) & $1.32(0.82, \mathrm{I} .52)$ & 0.315 & $1.56(0.62,1.73)$ & 0.358 \\
\hline Anesthesia time (>3h) & $1.39(0.66,2.93)$ & 0.395 & I.34(0.59,3.04) & 0.482 \\
\hline
\end{tabular}


Table 4 Multivariable Cox Proportional of OS and DFS

\begin{tabular}{|c|c|c|c|c|c|c|c|c|}
\hline \multirow[t]{2}{*}{ Variables } & \multicolumn{2}{|c|}{ OS (Before Matching) } & \multicolumn{2}{|c|}{ OS (After Matching) } & \multicolumn{2}{|c|}{ DFS (Before Matching) } & \multicolumn{2}{|c|}{ DFS (After Matching) } \\
\hline & HR $(95 \% \mathrm{Cl})$ & $P$-value & HR $(95 \% \mathrm{Cl})$ & $P$-value & HR $(95 \% \mathrm{Cl})$ & $P$-value & HR (95\% Cl) & $P$-value \\
\hline ERAS Protocol (no vs yes) & I.I8(1.07,2.02) & $<0.001$ & $1.13(1.02,2.12)$ & 0.013 & $1.80(1.53,2.71)$ & $<0.001$ & $1.46(1.32,2.62)$ & 0.016 \\
\hline Age (years) & $1.04(1.00,1.09)$ & 0.018 & & & $1.07(1.02,1.12)$ & 0.006 & & \\
\hline AFP $(>20 \mathrm{ng} / \mathrm{mL})$ & $1.57(1.08,2.11)$ & 0.022 & & & $1.25(0.84,1.85)$ & 0.267 & & \\
\hline Tumor encapsulation (None) & $2.06(0.76,3.46)$ & 0.157 & & & NA & & & \\
\hline Tumor staging (III-IV) & NA & & & & $1.12(0.76,1.66)$ & 0.576 & & \\
\hline Vascular invasion (Yes) & $2.55(1.34,4.86)$ & 0.004 & & & $1.89(1.25,2.84)$ & 0.002 & & \\
\hline ASA (III-IV) & $\mathrm{I} .75(1.04,2.56)$ & 0.039 & & & $2.34(1.04,3.39)$ & 0.045 & & \\
\hline
\end{tabular}

postoperative pain relief and early mobilization and organ function recovery, to ultimately promote patients' recovery, shorten hospital stay, reduce postoperative complications, and lessen health care costs. As mentioned, ERAS applied in general surgery achieved remarkable results. Our previous study found that implementation of ERAS programs can accelerate the recovery of patients who underwent radical cystectomy. ${ }^{15}$ Studies have shown that ERAS applied during the perioperative period of patients with hepatectomy for liver cancer was safe and effective. ${ }^{16,17}$ But there have been
A

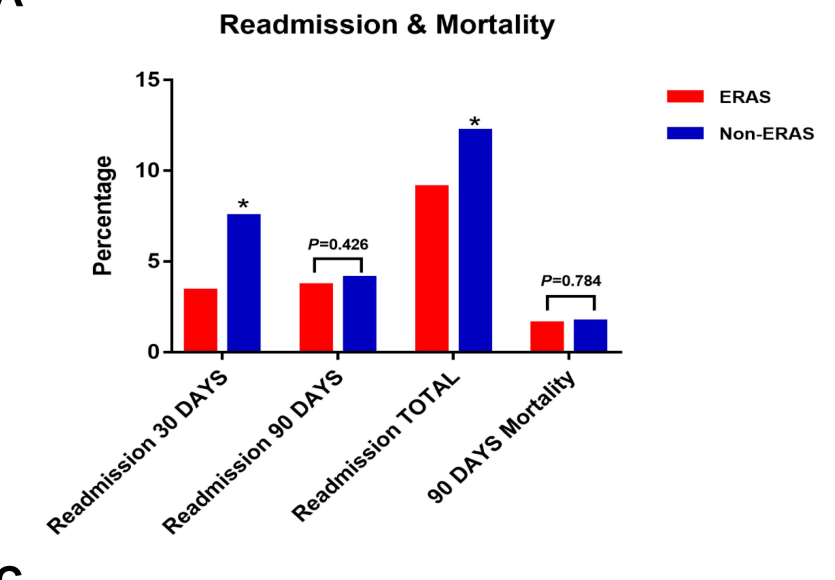

C

Clavien-Dindo Classification

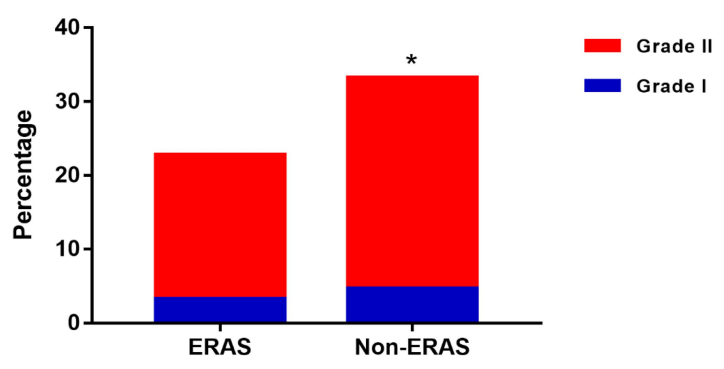

B

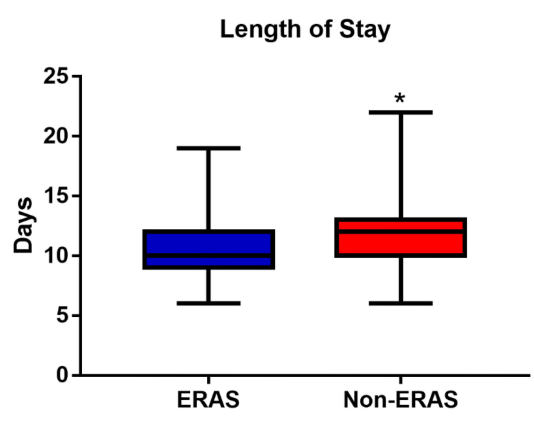

D Postoperative Complications

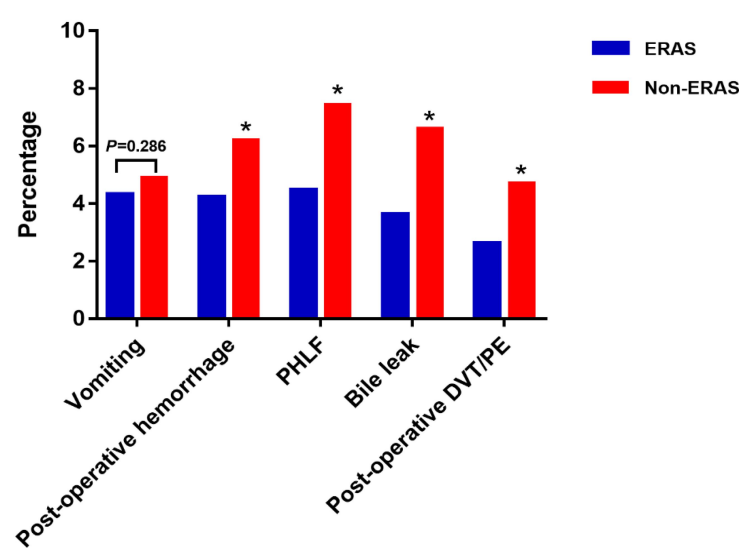

Figure 3 (A) Readmission rate and mortality between the ERAS group and non-ERAS group; (B) hospital length of stay between groups. (C) The percentage of postoperative complications between groups according to Clavien-Dindo classification; (D) postoperative complications between groups. *P<0.00I. 
few studies about the effect of ERAS on the long-term prognosis of patients who underwent hepatectomy. To our knowledge, this study is the first to date, evaluating the effect of enhanced recovery on long-term outcomes after hepatic resection by using propensity score matching.

This was a retrospective study. The method of propensity value matching analysis can effectively reduce the influence of confounders and selective deviations in retrospective studies, improve the statistical efficiency, and can effectively analyze non-random control data. ${ }^{18}$ PSM ensures the objectivity of the study, using similar covariate distributions to match the experimental and control groups without affecting the results of the study. In this study, the clinical baseline data of patients in the two groups were unbalanced before matching, and the difference of Child-Pugh and serum AFP between the two groups was statistically significant. After matching, the Child-Pugh and serum AFP were evenly distributed in the two groups, and the difference was not statistically significant, so the baseline data of patients in the two groups were more balanced.

Multiple observational studies supporting the benefits of ERAS have been increasing rapidly in recent years. ERAS significantly improves perioperative outcomes in different branches of surgery. ${ }^{19}$ The results of this study showed that the implementation of ERAS program in the perioperative period of hepatectomy patients with liver cancer could prolong the OS and DFS of the patients. Patients who underwent hepatectomy were prone to moderate and severe postoperative pain. ERAS advocates multimodal postoperative analgesia for better analgesia in postoperative pain treatment. In this study, the rate of moderate and severe postoperative pain in ERAS group was significantly lower than that in the non-ERAS group. Moreover, early feeding time, first postoperative exhaust time and time of getting out of bed in ERAS group were significantly earlier than in the non-ERAS group, which was basically the same as the previous study, suggesting that ERAS can significantly accelerate postoperative rehabilitation and speed up the recovery of postoperative intestinal function in patients. Studies have shown that ERAS, while applied to colorectal surgery, significantly improved short-term surgical outcomes in patients. ${ }^{8,20}$ In this study, the implementation of ERAS program decreased the 30-day readmission rate and readmission in total in patients who underwent hepatectomy, and the difference was statistically significant. Clavien-dindo classification system can easily and objectively reflect the overall severity of postoperative complications. ${ }^{21}$ The present study showed that the Clavien-dindo classification showed significant difference between the two groups. The postoperative LOS in ERAS group was significantly shorter than that of non-ERAS group, and the difference was statistically significant, indicating that the optimization measures of ERAS can speed up the postoperative recovery of patients. Postoperative complications (including postoperative hemorrhage, PHLF, bile leak, and postoperative DVT/PE), were significantly reduced in the ERAS group compared to the non-ERAS group. There was no difference in postoperative vomiting between the two groups. In this study, no patients in the two groups died during the perioperative period and were discharged smoothly. The results indicate that ERAS is safe and effective in the perioperative period of hepatectomy for liver cancer.

To our knowledge, there have been no studies evaluating long-term effects of ERAS in patients after hepatectomy for liver cancer up to now. In our study, significant differences were observed between the ERAS and the conservative group regarding short-term outcomes: length of hospital stay and postoperative complications, and long-term outcomes regarding overall survival and disease-free survival. A current study which assessed postoperative quality of life of 152 patients with a median follow-up of 48 months revealed preoperative ECOG status, surgeon experience, and daytime incontinence as independent prognostic features of a good postoperative quality of life. ${ }^{22}$ The impact of postoperative regimen on quality of life has not been examined so far. It is comprehensible that the postoperative management influences postoperative recovery in the short term and then influences long-term outcomes.

There were some limitations in the present study: this was a single-center study, no concurrent clinical control studies were conducted. Although the method of propensity matching analysis was used to eliminate some confounders and improve the statistical efficiency, the statistical method itself has some defects and the implementation of ERAS process may have affected the experimental results and cannot fully achieve the effect of randomized controlled trials (RCT). The clinical efficacy of ERAS in the perioperative period of hepatectomy for liver cancer needs to be explored by more large-scale multicenter prospective randomized controlled trials.

In summary, implementation of ERAS program in perioperative management could prolong OS and DFS of patients who underwent hepatectomy, reduce LOS, decrease the 30day postoperative readmission rate and 90-day postoperative mortality. It is feasible, safe, and effective, and of great significance in postoperative recovery in patients who underwent hepatectomy for liver cancer. 


\section{Disclosure}

The authors state that they have no conflicts of interest for this work.

\section{References}

1. Siegel RL, Miller KD, Jemal A. Cancer statistics, 2020. CA Cancer J Clin. 2020;70(1):7-30. doi:10.3322/caac.21590

2. Savikko J, Ilmakunnas M, Mäkisalo H, et al. Enhanced recovery protocol after liver resection. Br J Surg. 2015;102(12):1526-1532. doi:10.1002/bjs.9912

3. Hiller JG, Perry NJ, Poulogiannis G, et al. Perioperative events influence cancer recurrence risk after surgery. Nat Rev Clin Oncol. 2018;15(4):205-218. doi:10.1038/nrclinonc.2017.194

4. Kehlet H. Multimodal approach to control postoperative pathophysiology and rehabilitation. Br J Anaesth. 1997;78(5):606-617. doi:10. 1093/bja/78.5.606

5. Pal AR, Mitra S, Aich S, Goswami J. Existing practice of perioperative management of colorectal surgeries in a regional cancer institute and compliance with ERAS guidelines. Indian J Anaesth. 2019;63 (1):26-30. doi:10.4103/ija.IJA_382_18

6. Muehling B, Schelzig H, Steffen P, Meierhenrich R, SunderPlassmann L, Orend KH. A prospective randomized trial comparing traditional and fast-track patient care in elective open infrarenal aneurysm repair. World J Surg. 2009;33(3):577-585. doi:10.1007/ s00268-008-9892-2

7. Auyong DB, Allen CJ, Pahang JA, et al. Reduced length of hospitalization in primary total knee arthroplasty patients using an updated enhanced recovery after orthopedic surgery (ERAS) pathway. $J$ Arthroplasty. 2015;30(10):1705-1709. doi:10.1016/j.arth.2015.05. 007

8. Gonzalez-Ayora S, Pastor C, Guadalajara H, et al. Enhanced recovery care after colorectal surgery in elderly patients. Compliance and outcomes of a multicenter study from the Spanish working group on ERAS. Int J Colorectal Dis. 2016;31(9):1625-1631.

9. Xu Y, Liu A, Chen L, et al. Enhanced recovery after surgery (ERAS) pathway optimizes outcomes and costs for minimally invasive radical prostatectomy. J Int Med Res. 2020;48(6):300060520920072. doi:10. 1177/0300060520920072

10. Debono B, Sabatier P, Boniface G, et al. Implementation of enhanced recovery after surgery (ERAS) protocol for anterior cervical discectomy and fusion: a propensity score-matched analysis. Eur Spine J. 2020 .
11. Barbero M, García J, Alonso I, et al. ERAS protocol compliance impact on functional recovery in colorectal surgery. Cir Esp. 2020;99 (2):108-114. doi:10.1016/j.ciresp.2020.05.010

12. Aarts MA, Okrainec A, Glicksman A, et al. Adoption of enhanced recovery after surgery (ERAS) strategies for colorectal surgery at academic teaching hospitals and impact on total length of hospital stay. Surg Endosc. 2012;26(2):442-450. doi:10.1007/s00464-0111897-5

13. Qi S, Chen G, Cao P, et al. Safety and efficacy of enhanced recovery after surgery (ERAS) programs in patients undergoing hepatectomy: a prospective randomized controlled trial. J Clin Lab Anal. 2018;32 (6):e22434. doi:10.1002/jcla.22434

14. Delgado TC, Barbier-Torres L, Zubiete-Franco I, et al. Neddylation, a novel paradigm in liver cancer. Transl Gastroenterol Hepatol. 2018;3:37. doi:10.21037/tgh.2018.06.05

15. Zhang $\mathrm{H}$, Wang $\mathrm{H}$, Zhu $\mathrm{M}$, et al. Implementation of enhanced recovery after surgery in patients undergoing radical cystectomy: a retrospective cohort study. Eur J Surg Oncol. 2020;46(1):202-208. doi:10.1016/j.ejso.2019.07.021

16. Rouxel P, Beloeil H. Enhanced recovery after hepatectomy: a systematic review. Anaesth Crit Care Pain Med. 2019;38(1):29-34. doi:10.1016/j.accpm.2018.05.003

17. Fung AKY, Chong CCN, Lai PBS. ERAS in minimally invasive hepatectomy. Ann Hepatobiliary Pancreat Surg. 2020;24(2):119126. doi:10.14701/ahbps.2020.24.2.119

18. Benedetto U, Head SJ, Angelini GD, et al. Statistical primer: propensity score matching and its alternatives. Eur J Cardiothorac Surg. 2018;53(6):1112-1117. doi:10.1093/ejcts/ezy167

19. Rubinkiewicz M, Witowski J, Su M, et al. Enhanced recovery after surgery (ERAS) programs for esophagectomy. J Thorac Dis. 2019;11 (Suppl 5):S685-S691. doi:10.21037/jtd.2018.11.56

20. Kim MK, Kim J-G, Lee G, et al. Comparison of the effects of an ERAS program and a single-port laparoscopic surgery on postoperative outcomes of colon cancer patients. Sci Rep. 2019;9(1):11998. doi:10.1038/s41598-019-48526-1

21. Clavien PA, Barkun J, de Oliveira ML, et al. The Clavien-Dindo classification of surgical complications: five-year experience. Ann Surg. 2009;250(2):187-196. doi:10.1097/SLA.0b013e3181b13ca2

22. Kretschmer A, Grimm T, Buchner A, Stief CG, Karl A. Prognostic features for quality of life after radical cystectomy and orthotopic neobladder. Int Braz J Urol. 2016;42(6):1109-1120. doi:10.1590/ s1677-5538.ibju.2015.0491

\section{Publish your work in this journal}

Cancer Management and Research is an international, peer-reviewed open access journal focusing on cancer research and the optimal use of preventative and integrated treatment interventions to achieve improved outcomes, enhanced survival and quality of life for the cancer patient.
The manuscript management system is completely online and includes a very quick and fair peer-review system, which is all easy to use. Visit http://www.dovepress.com/testimonials.php to read real quotes from published authors. 